\title{
Development of a Versatile, Full-Featured Search Functionality for Indico
}

Penelope Constanta

CHEP 2019

4 November 2019
In collaboration with:

\section{BROOKHBVEN \\ NATIONAL LABORATORY}

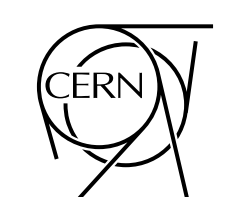




\section{The Collaboration}

- Fermilab

- Penelope Constanta

- BNL

- Ofer Rind

- Jose Caballero Bejar

- CERN

- Pedro Ferreira

- Adrian Mönnich

- Pablo Panero

- Carina Rafaela De Oliveira Antunes

- Aristofanis Chionis Koufakos 


\section{Overview}

- Indico is:

- an open-source event management system, popular in HEP community

- extensible through its plugin architecture (PayPal, video conferencing, search etc.)

- Indico v2.x:

- has many improvements throughout the system

- lacks search capabilities, outside the CERN eco-system that uses SharePoint

- Search plugin necessity:

- CERN is moving away from SharePoint by the end of this year, to the new invenio based CERN Search $\mu$ service, necessitating the development of an indico interface

- Fermi and BNL user communities requested a full functional search before deploying the new indico version

- Fermi-BNL-CERN collaboration to build the search plugins:

- Utilizing the new CERN Search $\mu$ Service and make it available to the community 


\section{Indico Search}

- Indico v0.98 - v1.2:

- search utilizes invenio (v1.1) as its search engine sending its metadata in XML format

- search results are formatted and displayed appropriately by indico

- Framework can be used outside CERN's environment

- Indico v1.9 - v2.2:

- search sends search metadata to SharePoint by re-purposing the existing invenio plugin code

- Metadata formatting does not take advantage of the new python packages (SQLAlchemy, marshmallow, etc.)

- search results are displayed by SharePoint (indico simply displays the SharePoint page)

- Framework cannot be used outside CERN's environment

- Collaboration plugin development for next version of indico v2.2.x:

- search utilizes invenio's (v3) CERN Search Api component and Elasticsearch as its search engine, sending its metadata in JSON format and taking advantage of SQLAlchemy and marshmallow

- search results are formatted and displayed appropriately by indico

- framework is developed so that it can be used outside CERN's environment 


\section{Indico Code Architecture}

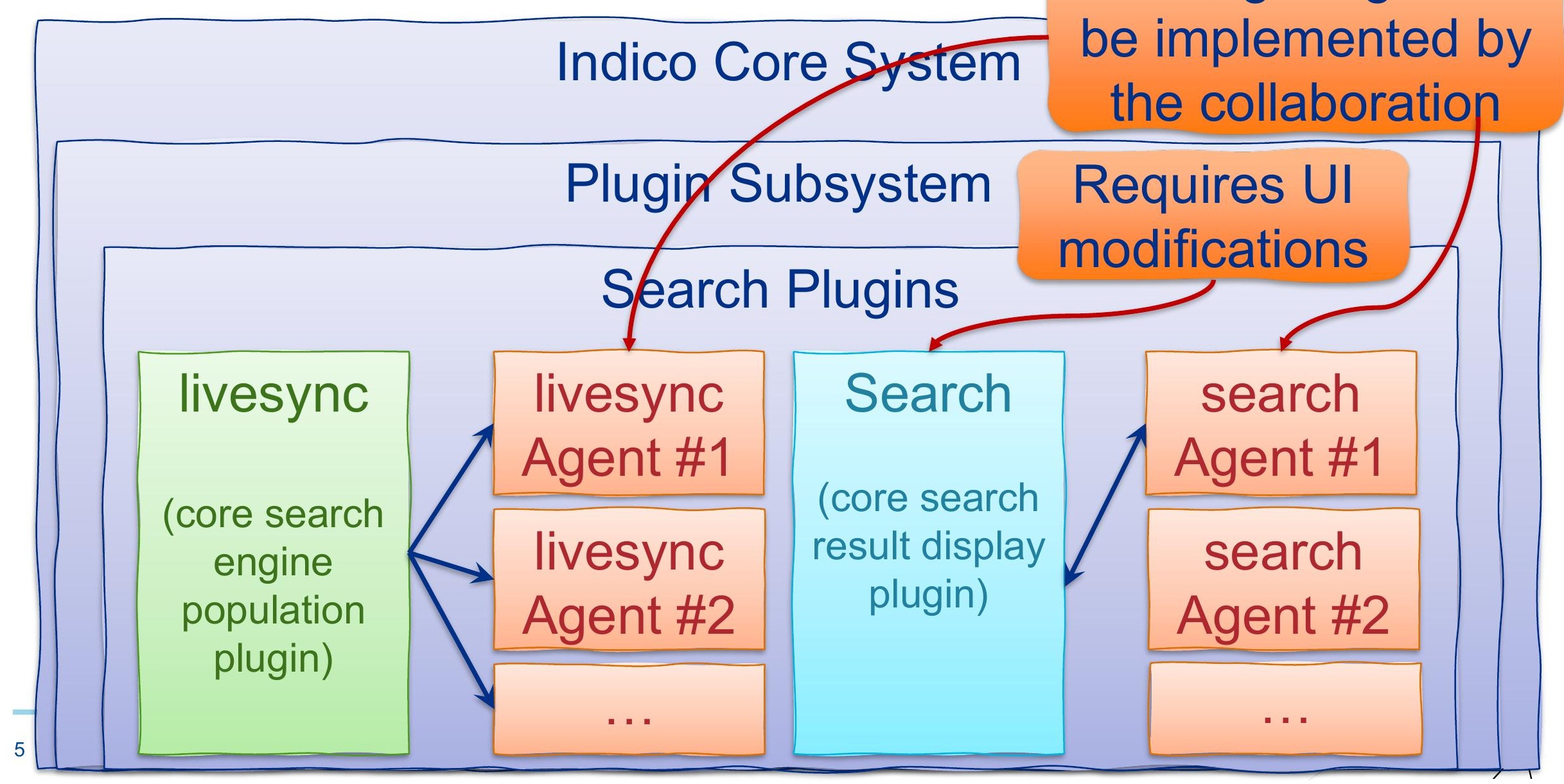

Missing Plugins to be implemented by the collaboration 
Indico Search System Architecture Sends indico object metadata to https://cernsearch_api/
Agent that sends search strings, receives \& displays search results (https://cernsearch_api/)

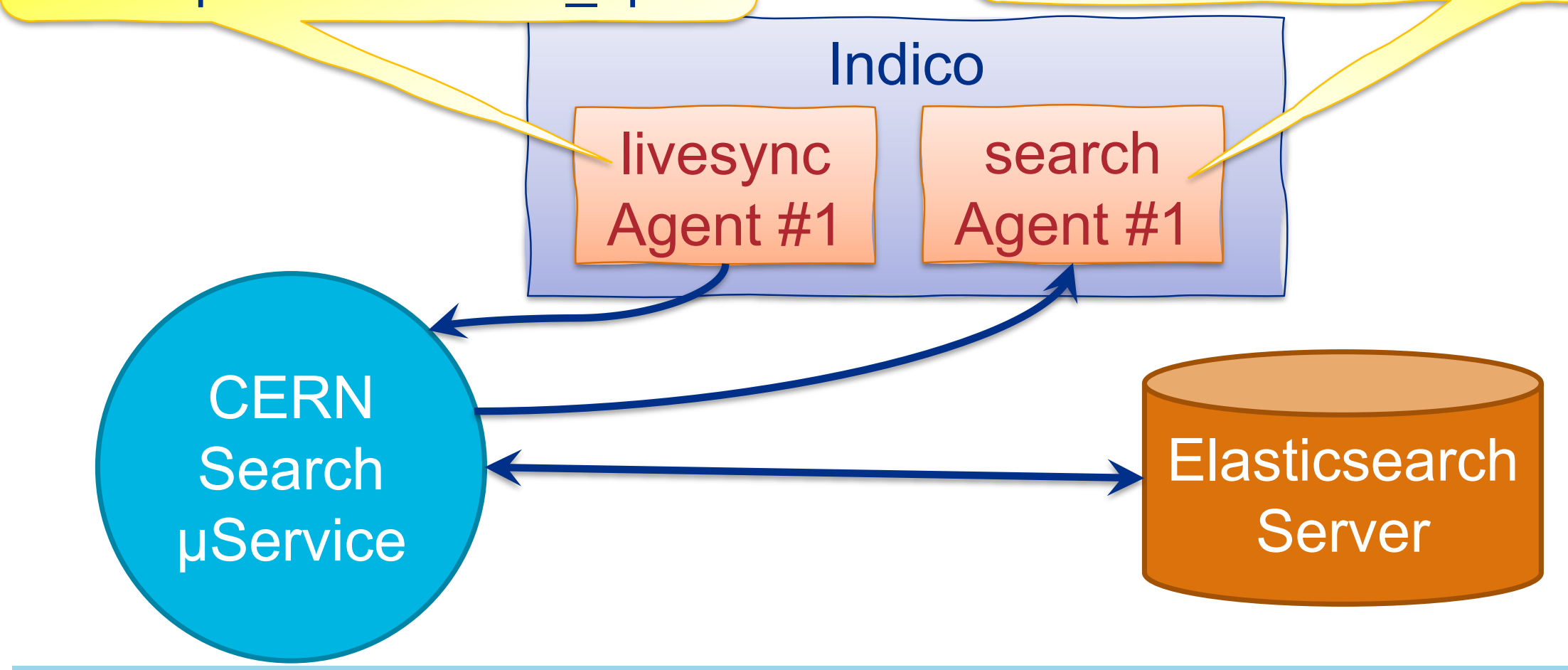




\section{Implementation Challenges}

- Indico v2.x moved away from the ZOPE database to PostgreSQL and almost the entire indico code was re-written and restructured

- Any familiarity with the previous versions' code is not useful

- Plugin development is seemingly easier but at the end one needs to understand all the internals of the new indico plugin system as well as the interface with the base plugins and the core indico code, along with the numerous new python packages

- CERN Search $\mu$ service is very new and documentation is targeted for CERN's internal use

- Deployment through docker-compose prove to be more challenging as the $\mu$ service is targeted for CERN's internal use.

- FNAL and BNL developers worked for a fraction of their time on the indico project and were not familiar with the used python packages. 


\section{Indico 2.x Installation / Configuration}

- CERN's documentation is excellent for installing/upgrading and setting up indico!

- Installation

- Just follow CERN's indico 2.x installation

- https://docs.getindico.io/en/latest/installation/production/

- For our development purposes we installed the developer's version:

- https://docs.getindico.io/en/latest/installation/development/

- Enable search plugins - Configuration

- All required steps are at:

- https://docs.getindico.io/en/latest/installation/plugins/ 


\section{Deployment of CERN Search $\mu$ service}

- CERN provided the docker-compose.yml that creates:

- The cern_search_rest_api as an invenio component

- The PostgreSQL application

- not required if connecting to an existing DB

- The Elasticsearch (ES) application

- not required if connecting to an existing ES installation

- The ES kibana application

- not required if connecting to an existing ES installation

- The tika server to parse PDF, pptx, LaTex etc. files, needs to be added, if not connecting to an existing tika server.

- It also initializes the invenio DB and uploads the ES mappings 


\section{Implementation Status}

- livesync agent for CERN Search uservice

- First version development almost completed

- Not fully tested, awaiting the cern-search-api deployment

- Indico search User Interface

- First version development completed, requires minor modifications

- Search results UI:

- provides filtering capabilities for Speakers and Affiliations

- uses different tabs for events, contributions, attachments, notes

- displayed page controls

- Tested with mock data

- search agent for CERN Search $\mu$ service

- Last stages of development

- Not fully tested, awaiting the cern-search-api deployment 


\section{Indico livesync Agent plugin Configuration}

LiveSync_Json is the agent for the CERN search uservice

indioD

Home Create event -

Room booking

Administration

My profile

Home " Administration " Plugins

General Settings

\section{Plugins}

(1) Security

† Plugins

? Room Booking

- 4. User Management

- \& Customization

Synchronization

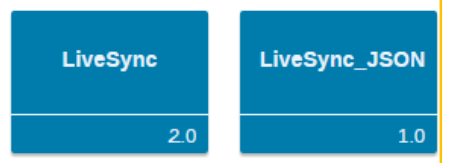

, Homepage

(4) Integration

(1) Tasks

(2)

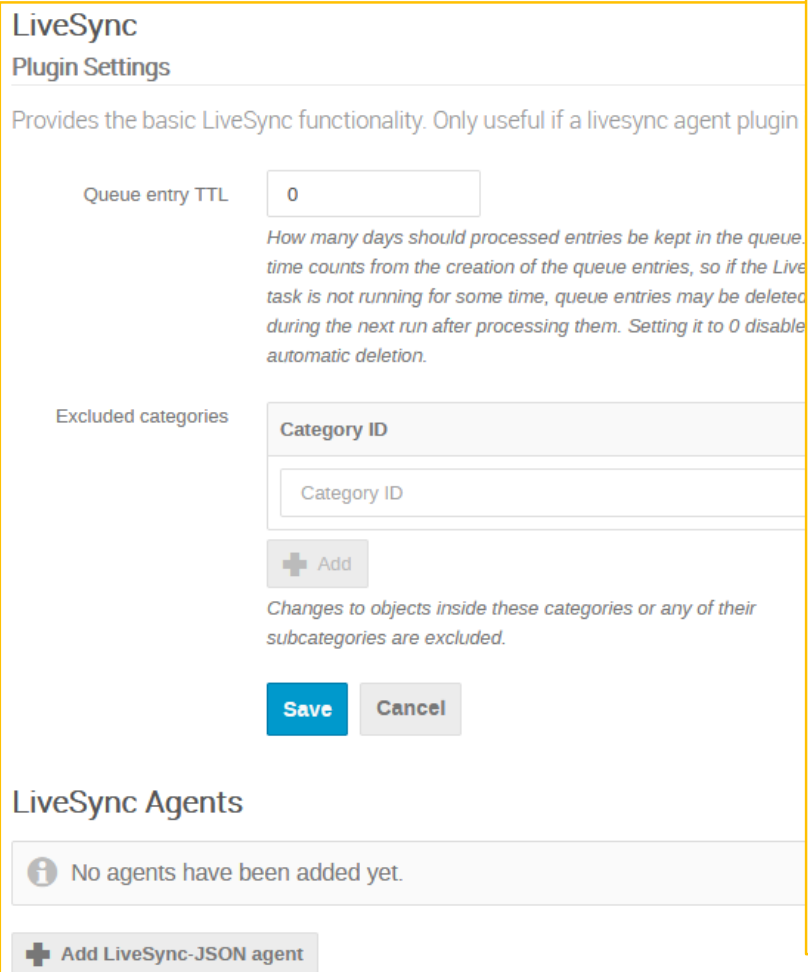

Category ID$$
\text { Category ID }
$$$$
4 \text { Add }
$$

Changes to objects inside these categories or any of their subcategories are excluded

Save

Cancel

LiveSync Agents

(i) No agents have been added yet.

4 Add LiveSync-JSON agent

LiveSync_JSON

Plugin Settings

Provides the LiveSync-JSON agent for LiveSync

Search app URL *

URL of search app import endpoint

Search app TOKEN *

TOKEN for accessing the Search app import endpoint

Elasticsearch Events *

JSON Schema

events_v1.1.0.json

the JSON Schema for the events Elasticsearch index

Elasticsearch * contributions_v1.1.0.json

Contributions JSON

Schem

the JSON Schema for the contributions Elasticsearch index

Elasticsearch *

SubContribution

subcontributions v1.1.0.json

JSON Schema

the JSON Schema for the subcontributions Elasticsearch index

Elasticsearch *

Attachments JSON Schema

atrachments_ v1.1.0.json

the JSON Schema for the attachments Elasticsearch index

Elasticsearch Notes

notes_v1.1.0.json

JSON Schema

the JSON Schema for the notes Elasticsearch index

tika server URL *

URL of tika server to parse file content. If not supplied a local tika server will be instantiated.

Save 


\section{Indico Search User Interface}

\section{Indice}

Home Create event

Room booking

Administration

Services -

My profile

Main categories

CHEP2019

(6) Public - (1) Europe/Zurich = 2 P. Ferreira -

Welcome to Indico. The Indico tool allows you to manage complex conferences, workshops and meetings. To start browsing, please select a category below.
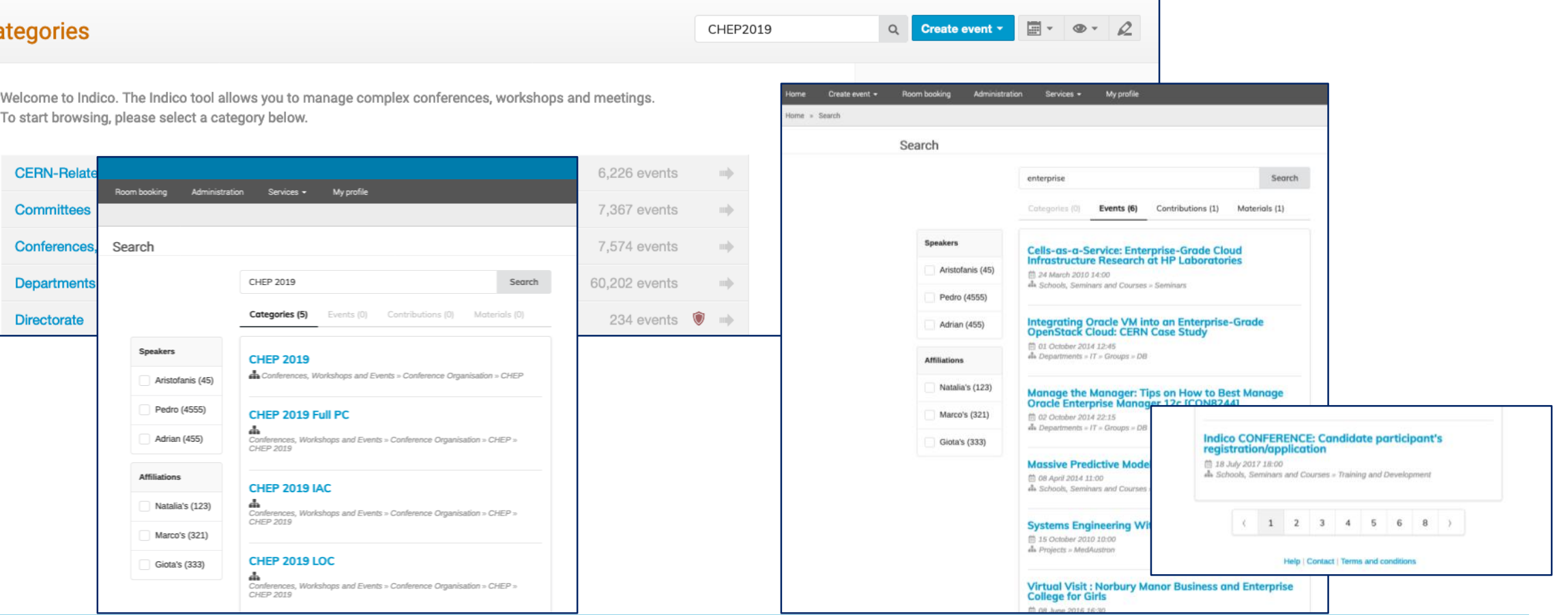


\section{Future Development}

- All plugins developed by the collaboration will be integrated into indico and CERN will take ownership.

- Further development may include:

- Improved resilience and recovery for the livesync agent

- Extensions to search UI, if needed

- Improved developer documentation and deployment for non-CERN environments

If you can find this talk on CERN's indico site, using indico search, in 2020 then this collaboration was successful! 